\title{
A Tutorial on Applications-Oriented Optimal Experiment Design
}

\author{
Cristian R. Rojas, Jonas Mårtensson and Håkan Hjalmarsson
}

\begin{abstract}
Presented in this chapter is a tutorial on the design of input signals for system identification. The chapter concerns the details of basic optimal experiment design, computational issues, implementation and conditions that lead to an optimal input signal. It is also addressed how the two fundamental issues associated with system identification, model structure identification and model validation relate to experiment design. Throughout the chapter, a simple FIR example is employed to illuminate the concepts presented.
\end{abstract}

\section{Introduction}

The problem of how to determine an adequate model from experimental data system identification - is a multi faceted topic, imposing quite high demands on the user. Two of the core issues are model structure selection and model validation. The key to simplify these problems and others lies in ensuring that suitable data is available for the modeling. What this means depends very much on what the model is going to be used for. We illustrate this with a simple example.

Example 1. Consider the finite impulse response (FIR) system

$$
y_{t}=\sum_{k=1}^{n} \theta_{k} u_{t-k}+e_{t}
$$

Cristian Rojas, Jonas Mårtensson and Håkan Hjalmarsson

ACCESS Linnaeus Center, School of Electrical Engineering, KTH Royal Institute of Technology, S-100 44 Stockholm, Sweden, e-mail: cristian.rojas@ee.kth.se,jonas.martensson@ee.kth.se,hakan.hjalmarsson@ee.kth.se 
where $y_{t}$ is the output, $u_{t}$ is the input and where $\left\{e_{t}\right\}$ is zero mean white noise with variance ${ }^{1} \lambda=\mathrm{E}\left[e_{t}^{2}\right]$, and where $\theta_{k} \in \mathbb{R}, k=1, \ldots, n$ are the impulse response coefficients.

Let $\theta=\left[\begin{array}{lll}\theta_{1} & \ldots & \theta_{n}\end{array}\right]^{T} \in \mathbb{R}^{n}$ and suppose that the objective is to estimate the frequency response $G\left(e^{j \omega}, \theta\right)=\sum_{k=1}^{n} \theta_{k} e^{-j \omega k}$ of the system. In particular let us assume that the frequency of interest is $\omega=0$, i.e. we are interested in the static gain $\sum_{k=1}^{n} \theta_{k}$ of the system. With a constant input $u_{t}=1, t=1, \ldots$, the system output will be

$$
y_{t}=\left(\sum_{k=1}^{n} \theta_{k}\right)+e_{t}
$$

i.e. the output will fluctuate around the sought after quantity making the estimation problem very simple regardless of the order of the system. However, with this input the individual impulse response coefficients $\left\{\theta_{k}\right\}_{k=1}^{n}$ cannot be identified and thus, e.g., the frequency response at other frequencies than 0 can not be recovered from the data.

Thus, a given data set may be suitable for one application but not for another. In this chapter we will outline how one can formulate optimal experiment design problems whereby the application is taken into account.

The bibliography on optimal experiment design is extensive and include the by now classical texts [14, 37, 2, 29]. The survey papers [1, 28] provide good overviews of the area. Recently there have been contributions to design in the frequency domain [15], set-membership identification [6], applications-oriented experiment design [18, 23, 19, 4], plant-friendly design [9], convexification of the associated optimization problems [18, 23], robust experiment design [34], least-costly identification where the cheapest experiment that fulfills given performance specifications is designed [8], and closed loop experiment design [20]. Also the robustness properties of optimal experiment designs have been highlighted [26]. The four $\mathrm{PhD}$ theses $[22,25,3,32]$ provide good coverage of recent developments.

In this tutorial we will follow the framework outlined in [19]. We will start in the next section by discussing how to formulate such problems as optimization problems. Next we will consider how to "translate" such optimization problems into a computationally tractable format. This is rooted in recent developments of convex optimization. It is well known that typically solutions to this type of problem depend on properties of the unknown system. Practical ways to handle this are discussed in Section 4. For applications where the amount of properties that have to be extracted from the system by way of system identification is limited it turns out that applications-oriented optimal experiment designs provide "added value". The way the estimated model affects the application becomes less sensitive to design choices in the identification step. This is discussed in Section 5.

\footnotetext{
${ }^{1} \mathrm{E}[x]$ denotes expectation of a random variable $x$.
} 
In order to not become submerged in technical details, we will keep the exposition simple by restricting ourselves to models of the type (1), estimated by way of standard least-squares.

\section{Applications-oriented experiment design}

\subsection{The set of acceptable models}

A simplified, but conceptually attractive way, to think of the objective of any modeling activity is that a model should be delivered to the user such that when it is used in the application, the resulting performance is acceptable. In fact, we could split the universe of all possible models into two sets: 1) $\mathscr{E}_{a p p}$ : the set of models for which the performance of the application is acceptable, and 2) the set complementary to $\mathscr{E}_{a p p}$, consisting of all unacceptable models. The optimal experiment design problem thus becomes that of designing an experiment such that it is ensured that the resulting model belongs to the set of acceptable models $\mathscr{E}_{\text {app }}$. How to achieve this depends on the underlying assumptions about the data generating mechanism, i.e. the system, the used model and the estimation method. Here, for simplicity, we will consider standard least-squares estimation.

How to quantify what acceptable performance means is of course application dependent. Here we will for simplicity assume that this is captured by that a scalar function is less than some given value $1 / \gamma$, we refer to Section 3.2 for details.

\subsection{A stochastic framework}

We will assume that data is generated by a system of the form (1). To simplify the discussion we will assume that the model structure is known, i.e. only the parameters $\theta=\left[\begin{array}{lll}\theta_{1} & \ldots & \theta_{n}\end{array}\right]^{T} \in \mathbb{R}^{n}$ are unknown. We will return to the situation when the structure is unknown in Section 5. With $\hat{\theta}_{N}$ denoting the parameter estimate based on $N$ input-output samples $\left\{y_{t}, u_{t}\right\}_{t=1}^{N}$, the source of the discrepancy between $\hat{\theta}_{N}$ and $\theta$ will then, neglecting unknown initial conditions, be due solely to the noise $\left\{e_{t}\right\}$ in (1). Assuming this quantity to be zero mean white noise implies that the error in the parameter estimate will be random. More specifically, the least-squares estimate of $\theta$ is given by [24]

$$
\hat{\theta}_{N}:=R_{N}^{-1} f_{N} \quad \text { where } \quad R_{N}:=\sum_{t=1}^{N} \varphi_{t} \varphi_{t}^{T}, \quad f_{N}:=\sum_{t=1}^{N} \varphi_{t} y_{t}
$$

where $\varphi_{t}=\left[\begin{array}{lll}u_{t-1} & \ldots & u_{t-n}\end{array}\right]^{T}$. Above, we have assumed that $R_{N}$ is full rank which corresponds to that data is persistently exciting [24]. Using (1), simple algebra gives 


$$
\hat{\theta}_{N}-\theta=R_{N}^{-1} \sum_{t=1}^{N} \varphi_{t} e_{t}
$$

Assuming the noise to be normal distributed, this implies that also the parameter estimate is normal distributed ${ }^{2}$

$$
\hat{\theta}_{N}-\theta \in N\left(0, \lambda R_{N}^{-1}\right)
$$

where $\lambda$ is the variance of the noise $\lambda=\mathrm{E}\left[e_{t}^{2}\right]$. From (3) it follows that $\left(\hat{\theta}_{N}-\right.$ $\theta)^{T} \frac{R_{N}}{\lambda}\left(\hat{\theta}_{N}-\theta\right)$ is $\chi^{2}$-distributed with $n$ degrees of freedom. With $\alpha$ denoting the 99.5\% percentile of this distribution, this in-turn implies that the parameter estimate will end up in the confidence ellipsoid

$$
\mathscr{E}_{i d}:=\left\{\bar{\theta}:(\bar{\theta}-\theta)^{T} \frac{R_{N}}{\lambda}(\bar{\theta}-\theta) \leq \alpha\right\}
$$

with $99.5 \%$ probability [24]. Notice that the ellipsoid $\mathscr{E}_{i d}$ depends on the experimental conditions since $R_{N}$ depends on the used input sequence.

\subsection{Stochastic applications-oriented experiment design}

With the set of acceptable models $\mathscr{E}_{a p p}$ given, one way of realizing the experiment design paradigm outlined in Section 2.1 is to design the input sequence such that

$$
\mathscr{E}_{i d} \subseteq \mathscr{E}_{a p p}
$$

since then with at least $99.5 \%$ probability, the model parameter estimate will belong to $\mathscr{E}_{\text {app }}$.

The condition (5) can be achieved by many different types of experiments and in order to make the problem well posed some criterion has to be introduced. In [8] it was proposed that experiment design should try to minimize the experimental cost. Following this, one should try to achieve (5) by as "cheap" an experiment as possible. This leads to the following input signal design problem

$$
\begin{aligned}
& \min _{u^{N}} V\left(u^{N}\right) \\
& \text { s.t. } \mathscr{E}_{i d} \subseteq \mathscr{E}_{\text {app }}
\end{aligned}
$$

where $u^{N}=\left[u_{1}, \ldots, u_{N}\right]^{T}$ and where the function $V\left(u^{N}\right)$ should measure the cost of the experiment.

To illustrate the approach we return to the frequency response estimation problem again.

${ }^{2} x \in N(m, P)$ denotes that the random variable $x$ is normal distributed with mean $m$ and covariance matrix $P$. 
Example 2 (Example 1 cont'd). The frequency response can be expressed as $G\left(e^{j \omega}, \theta\right)=$ $\theta^{T} \Gamma\left(e^{j \omega}\right)$ where $\Gamma\left(e^{j \omega}\right)=\left[e^{-j \omega} \ldots e^{-j \omega n}\right]^{T}, \omega \in \mathbb{R}$. Suppose that we want the squared error

$$
\left|G\left(e^{j \omega}, \hat{\theta}_{N}\right)-G\left(e^{j \omega}, \theta\right)\right|^{2}=\left(\hat{\theta}_{N}-\theta\right)^{T} \Gamma\left(e^{j \omega}\right) \Gamma^{*}\left(e^{j \omega}\right)\left(\hat{\theta}_{N}-\theta\right)
$$

( $x^{*}$ denotes the complex conjugate transpose of $x$ ) to be less than $1 / \gamma$ where the positive constant $\gamma$ represents the desired accuracy. This means that

$$
\mathscr{E}_{a p p}=\left\{\bar{\theta}:(\bar{\theta}-\theta)^{T} \Gamma\left(e^{j \omega}\right) \Gamma^{*}\left(e^{j \omega}\right)(\bar{\theta}-\theta) \leq \frac{1}{\gamma}\right\}
$$

\subsection{Alternative formulations}

The formulation (6) was introduced in [19] but there exist other problem formulations that aim at achieving a similar objective. Here we briefly outline an approach that is closely connected to so called $L$-optimal design. We continue to use the frequency function estimation problem as example. The frequency function estimate is given by $G\left(e^{j \omega}, \hat{\theta}_{N}\right)=\hat{\theta}_{N}^{T} \Gamma\left(e^{j \omega}\right)$, which, being linear in $\hat{\theta}_{N}$, has variance

$$
\mathrm{E}\left[\left|G\left(e^{j \omega}, \hat{\theta}_{N}\right)-G\left(e^{j \omega}, \theta\right)\right|^{2}\right]=\Gamma^{*}\left(e^{j \omega}\right) R_{N}^{-1} \Gamma\left(e^{j \omega}\right)
$$

due to (3). Thus the constraint

$$
\Gamma^{*}\left(e^{j \omega}\right) R_{N}^{-1} \Gamma\left(e^{j \omega}\right) \leq \beta
$$

for some suitably chosen constant $\beta$, captures that a certain quality of the estimate of $G\left(e^{j \omega}, \theta\right)$ is required. Replacing the set constraint in (6) by (10) gives

$$
\begin{aligned}
& \min _{u^{N}} V\left(u^{N}\right) \\
& \text { s.t. } \Gamma^{*}\left(e^{j \omega}\right) R_{N}^{-1} \Gamma\left(e^{j \omega}\right) \leq \beta
\end{aligned}
$$

We refer to [23] for further details on this approach.

\section{Computational aspects}

When solving the input signal design problems (6) and (11) it is often more convenient to do it in the frequency domain. Below we will show that we can cast both these problems as convex programs in this domain. 


\subsection{Choice of decision variables}

It is the matrix $R_{N}$ in (6) and (11) that depends on the input. However, using the input sequence directly as decision variable leads to a non-convex problem. Turning to the frequency domain, the discrete Fourier transform (DFT) [27] of the input sequence is given by

$$
U\left(e^{j \mu_{k}}\right):=\sum_{t=1}^{N} u_{t} e^{-j \mu_{k} t}, \quad \mu_{k}=2 \pi k / N, \quad k=1, \ldots, N
$$

and with a periodic extension of the input, the DFT of the regressor sequence $\left\{\varphi_{t}\right\}$ is given by $\sum_{t=1}^{N} \varphi_{t} e^{-j \mu_{k} t}=\Gamma\left(e^{j \mu_{k}}\right) U\left(e^{j \mu_{k}}\right)$. By Parseval's theorem, [27], the matrix $R_{N}$ can be expressed as

$$
R_{N}=\sum_{t=1}^{N} \varphi_{t} \varphi_{t}^{T}=\frac{1}{N} \sum_{k=1}^{N} \Gamma\left(e^{j \mu_{k}}\right)\left|U\left(e^{j \mu_{k}}\right)\right|^{2} \Gamma^{*}\left(e^{j \mu_{k}}\right)
$$

Now we let the input be parameterized by the coefficients $\left\{c_{t}\right\}_{t=0}^{N-1}$ as

$$
\left|U\left(e^{j \mu_{k}}\right)\right|^{2}=\sum_{t=0}^{N-1} c_{t} e^{-j \mu_{k} t}
$$

Then the $(p, q)$-element of $R_{N}$ is equal to $c_{|p-q|}$. Thus $R_{N}$ is linearly parametrized in terms of these cofficients. As we will see below this is instrumental in order to convexify both (6) and (11).

Since $\left|U\left(e^{j \mu_{k}}\right)\right|^{2} \geq 0$, it has to hold that

$$
\sum_{t=0}^{N-1} c_{t} e^{-j \mu_{k} t} \geq 0, \quad k=1, \ldots, N
$$

for $\left\{c_{t}\right\}_{t=0}^{N-1}$ to be a valid parametrization. One implication of this is that $c_{N-j}=c_{j}$. These constraints thus have to be included.

\subsection{Using the set of acceptable models}

Let us now turn our attention to the set of acceptable models $\mathscr{E}_{\text {app }}$. Consider first the frequency function estimation example.

Example 3 (Example 2 cont'd). As we saw in (4) the confidence set $\mathscr{E}_{i d}$ is an ellipsoid.

Furthermore, since (8) is quadratic in $\bar{\theta}, \mathscr{E}_{a p p}$ for the frequency response estimation problem also is an ellipsoid ${ }^{3}$. It is easy to verify, see [19], that the set constraint

${ }^{3}$ To be more precise it is a degenerate ellipsoid since $\Gamma\left(e^{j \omega}\right) \Gamma^{*}\left(e^{j \omega}\right)$ is singular 
$\mathscr{E}_{i d} \subseteq \mathscr{E}_{\text {app }}$ is equivalent to

$$
R_{N}-\gamma \lambda \alpha \Gamma\left(e^{j \omega}\right) \Gamma^{*}\left(e^{j \omega}\right) \geq 0
$$

Now, since $R_{N}$ is linear in the decision variables $\left\{c_{t}\right\}_{t=0}^{N-1}$, this inequality is a linear matrix inequality (LMI) which is a convex constraint. If $V\left(u^{N}\right)$ is the input energy, i.e. $V\left(u^{N}\right)=\sum_{t=1}^{N} u_{t}^{2}$ which in turn, using Parseval's theorem, can be written

$$
V\left(u^{N}\right)=c_{0}
$$

then the optimal input signal design problem (6) can be written

$$
\begin{aligned}
\min _{c_{0}, \ldots, c_{N-1}} & c_{o} \\
\text { s.t. } & {\left[\begin{array}{ccccc}
c_{o} & c_{1} & & \ldots & c_{N-1} \\
c_{1} & c_{0} & c_{1} & \ldots & c_{N-2} \\
\vdots & \ddots & \ddots & \ddots & \vdots \\
c_{N-2} & c_{N-1} & \ddots & \ddots & c_{1} \\
c_{N-1} & c_{N-2} & \ldots & c_{1} & c_{0}
\end{array}\right]-\gamma \lambda \alpha \Gamma\left(e^{j \omega}\right) \Gamma^{*}\left(e^{j \omega}\right) \geq 0 } \\
& \sum_{t=0}^{N-1} c_{t} e^{-j \mu_{k} t} \geq 0, \quad k=1, \ldots, N \\
& c_{N-j}=c_{j}, \quad j=1, \ldots, N-1
\end{aligned}
$$

which is a semi-definite program and hence convex.

For example for $\omega=0$, i.e. the static gain estimation problem discussed in Example 1 , we have

$$
\begin{aligned}
\min _{c_{0}, \ldots, c_{N-1}} & c_{o} \\
\text { s.t. } & {\left[\begin{array}{ccccc}
c_{o} & c_{1} & & \ldots & c_{N-1} \\
c_{1} & c_{0} & c_{1} & \ldots & c_{N-2} \\
\vdots & \ddots & \ddots & \ddots & \vdots \\
c_{N-2} & c_{N-1} & \ddots & \ddots & c_{1} \\
c_{N-1} & c_{N-2} & \ldots & c_{1} & c_{0}
\end{array}\right]-\gamma \lambda \alpha\left[\begin{array}{cccc}
1 & 1 & \ldots & 1 \\
1 & 1 & \ldots & 1 \\
\vdots & \vdots & & \vdots \\
1 & 1 & \ldots & 1 \\
1 & 1 & \ldots & 1
\end{array}\right] \geq 0 } \\
& \sum_{t=0}^{N-1} c_{t} e^{-j \mu_{k} t} \geq 0, \quad k=1, \ldots, N \\
& c_{N-j}=c_{j}, \quad j=1, \ldots, N-1
\end{aligned}
$$

The diagonal elements in the matrix inequality give $c_{0} \geq \gamma \lambda \alpha$. However, then it is straightforward to see that the choice $c_{0}=c_{1}=\ldots=c_{N-1}=\gamma \lambda \alpha$ both satisfies the matrix inequality and the positivity constraints (24). This solution corresponds to 


$$
\left|U\left(e^{j \mu_{k}}\right)\right|^{2}= \begin{cases}N \gamma \lambda \alpha & k=N \\ 0 & k=1, \ldots, N-1\end{cases}
$$

which in turn (taking the inverse DFT) corresponds to

$$
u_{t}=\sqrt{\gamma \lambda \alpha}, \quad t=1, \ldots, N
$$

Thus we have proven that a constant input signal is optimal when the static gain is the quantity of interest.

In general, $\mathscr{E}_{\text {app }}$ may not be convex. One can then use the following approximation. Consider a set of acceptable models given by

$$
\mathscr{E}_{a p p}=\left\{\bar{\theta}: V_{a p p}(\bar{\theta}) \leq \frac{1}{2 \gamma}\right\}
$$

for some function $V_{a p p}(\bar{\theta})$ that has a global minimum equal to zero at the true parameter value $\theta$. A second order approximation is then given by

$$
V_{a p p}(\bar{\theta}) \approx \frac{1}{2}(\bar{\theta}-\theta)^{T} V_{a p p}^{\prime \prime}(\theta)(\bar{\theta}-\theta)
$$

since the gradient $V_{a p p}^{\prime}(\theta)$ is zero by construction. Also, by construction, the second derivative $V_{a p p}^{\prime \prime}(\theta)$ is positive definite at the global optimum and when the approximation (27) is used in (26) the set of acceptable models is an ellipsoid given by

$$
\mathscr{E}_{a p p} \approx\left\{\bar{\theta}:(\bar{\theta}-\theta)^{T} V_{a p p}^{\prime \prime}(\theta)(\bar{\theta}-\theta) \leq \frac{1}{\gamma}\right\}
$$

Following the reasoning in Example (3) gives that the condition $\mathscr{E}_{\text {id }} \subseteq \mathscr{E}_{\text {app }}$ (5) can be approximated by

$$
R_{N}-\gamma \lambda \alpha V_{a p p}^{\prime \prime}(\theta) \geq 0
$$

\subsection{Using variance constraints}

Now we turn to the alternative constraint formulation (10) which involves the inverse of $R_{N}$. By making use of Schur complements, see e.g. [38], it can be translated to a constraint that is linear in $R_{N}$. Consider the partitioned hermitian matrix $M=\left(\begin{array}{cc}A & B \\ B^{*} & C\end{array}\right)$ where $A$ is square and non-singular. The Schur complement of $A$ in $M$ is defined as $S_{A}:=C-B^{*} A^{-1} B$ and it holds that $M \geq 0$ if and only if $A>0$ and $S_{A} \geq 0$. The constraint (11) can be written as $\beta-\Gamma^{*}\left(e^{j \omega}\right) R_{N}^{-1} \Gamma\left(e^{j \omega}\right) \geq 0$ where the left-hand side is the Schur complement of $R_{N}$ in the partitioned matrix $M_{R}:=\left(\begin{array}{cc}R_{N} & \Gamma\left(e^{j \omega}\right) \\ \Gamma\left(e^{j \omega}\right)^{*} & \beta\end{array}\right)$. Since $R_{N}>0$ by assumption it follows that (11) is equivalent to $M_{R} \geq 0$. Using the same arguments again we find that $M_{R} \geq 0$ if and only 
if the Schur complement of $\beta$ in $M_{R}$ is positive semi-definite ( $\beta$ is positive by assumption):

$$
R_{N}-\frac{1}{\beta} \Gamma\left(e^{j \omega}\right) \Gamma^{*}\left(e^{j \omega}\right) \geq 0
$$

Note that, with $\beta=\frac{1}{\lambda \alpha \gamma}$, (30) is also equivalent to the constraint (29) with $V_{a p p}\left(\hat{\theta}_{N}\right)$ given by (7). Thus we end up with exactly the same optimization problem (18)-(21) as before, but with $\beta$ replacing $\frac{1}{\lambda \alpha \gamma}$. We refer to [23] for generalizations.

\section{How to handle system dependency of the optimal solution}

In the formulation (6), typically either $\mathscr{E}_{i d}$ or $\mathscr{E}_{a p p}$ (or both) depend on the true system, i.e., we have $\mathscr{E}_{i d}=\mathscr{E}_{i d}(\theta)$ and $\mathscr{E}_{a p p}=\mathscr{E}_{a p p}(\theta)$. This usually implies that the optimal input signal will be a function of something which is unknown prior to the experiment.

In our FIR example, $\mathscr{E}_{i d}$ is independent of $\theta$, since it is a linearly parameterized model structure (unlike, e.g., ARX or ARMAX structures, where the covariance does depend on $\theta$ ). For the frequency estimation problem, $\mathscr{E}_{a p p}$ is also independent of $\theta$. However, if we were interested instead in estimating the squared magnitude of the frequency function at a given frequency, $\left|G\left(e^{j \omega}\right)\right|^{2}$, then a Taylor expansion shows that $\mathscr{E}_{a p p}$ is approximately

$$
\begin{aligned}
\mathscr{E}_{\text {app }}(\theta) & =\left\{\bar{\theta}:\left(\left|G\left(e^{j \omega}, \bar{\theta}\right)\right|^{2}-\left|G\left(e^{j \omega}, \theta\right)\right|^{2}\right)^{2} \leq \frac{1}{\gamma}\right\} \\
& \approx\left\{\bar{\theta}:(\bar{\theta}-\theta)^{T} \Gamma\left(e^{j \omega}\right)\left|G\left(e^{j \omega}, \theta\right)\right|^{2} \Gamma^{*}\left(e^{j \omega}\right)(\bar{\theta}-\theta) \leq \frac{1}{4 \gamma}\right\}
\end{aligned}
$$

where the dependence on $\theta$ through $\left|G\left(e^{j \omega}, \theta\right)\right|^{2}$ is clear.

The fact that the optimal input signal is a function of the true plant is not necessarily a problem if we already had some prior information, say from a previous experiment, and our goal is to design a new experiment to improve an existing model. In this case, we can simply replace in the formulation (6) $\mathscr{E}_{i d}$ and $\mathscr{E}_{a p p}$ by $\mathscr{E}_{i d}(\hat{\theta})$ and $\mathscr{E}_{\text {app }}(\hat{\theta})$, respectively, where $\hat{\theta}$ is a basic estimate of the plant; the input signal obtained in this way is called a locally optimal input signal design [13].

However, there are situations where we do not have enough reliable prior information to design our input signal based on a previous estimate of the plant. In the following subsections we describe two approaches to overcome this difficulty. 


\subsection{Robust designs}

Even in the absence of a preliminary estimate of the plant, we might have some basic knowledge about its parameters. For example, from physical considerations it is sometimes possible to infer the order of magnitude of the dominant time constant, the static gain, etc. If this is the case, we can assume that we know a priori a set $\Theta$ such that $\theta \in \Theta$, and reformulate the optimal input signal design problem (6) to account for this information. One possibility is to consider the following robust version of (6):

$$
\begin{aligned}
& \min _{u^{N}} V\left(u^{N}\right) \\
& \text { s.t. } \mathscr{E}_{i d}(\theta) \subseteq \mathscr{E}_{a p p}(\theta), \text { for all } \theta \in \Theta
\end{aligned}
$$

This problem corresponds to a robust convex program, which, save for a few exceptions, cannot be solved exactly in a computationally tractable way [7]. Here we present a simple approximate method to solve (31), based on a probabilistic relaxation technique known as the scenario approach [10]. For details, the reader is referred to [35]. The basic idea is to replace (31) by

$$
\begin{aligned}
& \min _{u^{N}} V\left(u^{N}\right) \\
& \text { s.t. } \mathscr{E}_{i d}\left(\theta_{i}\right) \subseteq \mathscr{E}_{a p p}\left(\theta_{i}\right), \quad i=1, \ldots, m
\end{aligned}
$$

where $\theta_{1}, \ldots, \theta_{m}$ are independent random samples from a distribution $P_{\theta}$ in $\Theta$. In [11] it was shown that if (32) is formulated in terms of $d$ decision variables and $m$ is chosen such that

$$
\left(\begin{array}{l}
m \\
d
\end{array}\right)(1-\varepsilon)^{m-d}<\delta
$$

for some constants $\delta, \varepsilon>0$, then the solution of (32) will, with probability greater than $1-\delta$, satisfy 'most' of the constraints in (31). More precisely, $P_{\theta}(\{\theta \in \Theta$ : $\left.\left.\mathscr{E}_{i d}(\theta) \nsubseteq \mathscr{E}_{\text {app }}(\theta)\right\}\right)<\varepsilon$ holds with probability $1-\delta$.

In Corollary 1 in [10], the following more explicit (but conservative) expression for $m$ was proposed: $m \geq(2 / \varepsilon) \ln (1 / \delta)+2 d+(2 d / \varepsilon) \ln (2 / \varepsilon)$. For example, for $d=30, \varepsilon=0.01$ and $\delta=10^{-10}, m$ should be at least 7864 , which gives a tractable semidefinite program (solvable within 10 minutes on a computer with Intel Core 2 Duo CPU of $2.53 \mathrm{GHZ}$ and a standard SDP solver)."

\subsection{Adaptive and sequential designs}

The idea of designing an experiment based on a previous estimate, and then redesigning the experiment from the previous one can be carried out even further, to 
Fig. 1 Adaptive input signal

design.

arrive at the concept of adaptive input signal design. In this approach, a recursive estimator is employed to obtain a new estimate of the plant at each time instant, and the recursive estimate is then used to simultaneously redesign the input sequence. Figure 1 presents a block diagram of the adaptive technique.

As explained in [16], adaptive input signal design can achieve optimal performance (i.e., the same performance as if the experiment were designed based on the knowledge of the true system) under mild conditions, even though the convergence proofs are difficult and beyond the scope of the present tutorial. The interested reader is referred to $[16,17]$ for details, in particular the latter reference contains a convergence proof for adaptive signal design for ARX models.

\section{Added value of optimal input signal design}

In the previous sections we have focused on the formulation of applications-oriented optimal input signal design problems and how to solve the associated optimization problems. An obvious question is how much that can be gained by optimal input signal design. The answer to this question is clearly problem dependent, and also depends on what one compares with. The reduction in experiment time in the two case studies (a process control application and a mechanical system) presented in [5] was roughly a factor 4 as compared to a Pseudo-Random Binary Sequence. The paper [12] presents results from re-tuning of an MPC controller of a process having 34 inputs and 90 outputs at one of the world's largest refineries, the Hovensa refinery in Virgin Islands, United States. Partially through the use of optimal experiment design, the total modeling time was reduced by $90 \%$.

By solving the optimal applications-oriented input signal design problem for different model complexities and different performance specifications it is possible to characterize how the minimal experimental cost depends on these quantities. This cost of complexity quantification, as it has become known, may provide valuable information for trading off performance in the application versus experimental effort in the identification problem. In Section 5.1 we discuss this for our standing example.

It is fairly obvious that optimal input signal design helps make system properties of importance easy to detect in the measured signals. However, a less obvious mechanism is that the optimal input signal tries to avoid to excite system behaviours that are inessential for the application. Thus it hides properties which are not important to the user. This dual aspect of the input signal has some very pleasant consequences, e.g.

1. It keeps the cost of the experiment at a reasonable level for models of high order. 
2. It sometimes allows for consistent estimation of the properties of interest, even in the presence of unmodelled dynamics.

We will discuss this further in Section 5.2.

\subsection{Cost of complexity}

By focusing on specific properties of a given system, it is possible to reduce the identification effort, measured in terms of the input signal energy required to estimate the desired property within a given accuracy.

Example 4 (Example 3 cont'd). Suppose that (8) is modified to

$$
\mathscr{E}_{a p p}=\left\{\bar{\theta}:(\bar{\theta}-\theta)^{T} \Gamma\left(e^{j \omega}\right) \Gamma^{*}\left(e^{j \omega}\right)(\bar{\theta}-\theta) \leq \frac{1}{\gamma}, \forall \omega \in\left[0, \omega_{B}\right]\right\},
$$

i.e. it is required that the accuracy of the frequency response is $\gamma$ over the frequency range $\left[0, \omega_{B}\right]$, where $0<\omega_{B} \leq \pi$. Then, following [33], it can be shown that the minimum input signal energy required (for large model orders $n$ ) is approximately

$$
n \lambda \gamma \omega_{B}
$$

where $\lambda$ is the variance of the noise $e_{t}$ and $1 / \gamma$ is the maximum allowed variance of the frequency function estimate in the range $\left[0, \omega_{B}\right]$.

According to (34), the required input signal energy increases in proportion to the model order $n$. However, this cost can be kept low by focusing on a more specific frequency region, i.e., by reducing $\omega_{B}$. This observation reenforces the statement that input signal design can hide those properties which are unimportant, thus providing an energy efficient experiment.

As a comparison, it can be shown, see [33] for details, that using a PRBS (Pseudo Random Binary Sequence) as input signal requires the energy $n \lambda \gamma \pi$ regardless of the bandwidth $\omega_{B}$ to ensure that the parameter estimate ends up in (33). Thus, for large model orders $n$, the reduction in required input energy through the use of optimal input signal design can be significant for small to moderate bandwidths $\omega_{B}$, as compared to PRBS excitation.

\subsection{Consistent estimation with unmodelled dynamics}

A simple example of the phenomenon that sometimes optimal input signal design allows the use of restricted models is given by the static gain estimation problem studied in the examples of this chapter.

Example 5 (Example 4 cont'd). In Example 3 we saw that a constant input signal is optimal for the problem of estimating the static gain. However, this particular input 
signal has the additional property that it still allows for consistent estimation of the static gain if we use the following simple static model: $y_{t}=K u_{t}$. The reason lies in the fact that a constant input signal completely hides all the other properties of the system by making them unidentifiable, This since, after the transient has died out, the system behaves as

$$
y_{t}=\left(\sum_{k=1}^{n} \theta_{k}\right) u_{t}+e_{t}
$$

when $u_{t}=u$ (constant).

A perhaps more interesting example is given in [25], where it has been shown that a nonminimum phase zero of a system $G$ (of arbitrary order) at $z=z_{o}$ can be consistently estimated with a model of the form $y_{t}=b_{1} u_{t-1}+b_{2} u_{t-2}$ by using an input signal

$$
u_{t}=\frac{1}{1-z_{o}^{-1}} r_{t}
$$

where $r_{t}$ is zero mean white noise. This input signal depends on the property of interest, but it can be implemented using the adaptive scheme of Section 4.2 [31].

\section{Concluding remarks}

We have in this chapter provided the basics of applications-oriented experiment design. There exists a wide range of extensions. By viewing the input signal as a stationary signal with a continuous spectral density $\Phi_{u}\left(e^{j \mu}\right)$, the spectral density takes the place of $\left|U\left(e^{j \mu_{k}}\right)\right|^{2}$ in the formulation above and the discrete-time Fourier transform (DTFT) is used instead of the DFT. An input signal with the desired spectral density can be generated by filtering white noise through the spectral factor of $\Phi_{u}(z)$. The Kalman-Yakubovich-Popov lemma $[36,30]$ can be used to replace the positivity condition on the input spectrum by a linear matrix inequality. This allows a wide range of model structures to be used, e.g. ARX, ARMAX, output-error and Box-Jenkins models. We refer to [22] for details. Also closed loop experiment design can be handled in this way [20] and there is emerging work on applicationsoriented experiment design for non-linear systems [21].

\section{References}

1. Atkinson, A., Bailey, R.: One hundred years of the design of experiments on and off the pages of Biometrika. Biometrika 88, 53-97 (2001)

2. Atkinson, A., Doner, A.: Optimum experiment design. Clarendon Press, Oxford (1992) 
3. Barenthin, M.: Complexity issues, validation and input design for control in system identification. Doctoral thesis, KTH, Stockholm, Sweden (2008)

4. Barenthin, M., Bombois, X., Hjalmarsson, H., Scorletti, G.: Identification for control of multivariable systems: Controller validation and experiment design via LMIs. Automatica 44(12), 3070-3078 (2008)

5. Barenthin, M., Jansson, H., Hjalmarsson, H.: Applications of mixed $\mathscr{H}_{\infty}$ and $\mathscr{H}_{2}$ input design in identification. In: 16th World Congress on Automatic Control. IFAC, Prague, Czech Republik (2005). Paper Tu-A13-TO/1

6. Belforte, G., Gay, P.: Optimal input design for set-membership identification of Hammerstein models. International Journal of Control 76(3), 217-225 (2003)

7. Ben-Tal, A., Nemirovski, A.: Robust convex optimization. Mathematics of Operations Research 23(4), 769-805 (1998)

8. Bombois, X., Scorletti, G., Gevers, M., Van den Hof, P.M.J., Hildebrand, R.: Least costly identification experiment for control. Automatica 42(10), 1651-1662 (2006)

9. Braun, M., Ortiz-Mojica, R., Rivera, D.: Application of minimum crest factor multisinusoidal signals for "plant-friendly" identification of nonlinear process systems. Control Engineering Practice 10(3), 301-313 (2002)

10. Calafiore, G.C., Campi, M.C.: The scenario approach to robust control design. IEEE Transactions on Automatic Control 51(5), 742-753 (2006)

11. Campi, M.C., Garatti, S.: The exact feasibility of randomized solutions of uncertain convex programs. SIAM Journal on Optimization 19(3), 1211-1230 (2008)

12. Celaya, P., Tkatch, R., Zhu, Y., Patwardhan, R.: Closed-loop identification at the Hovensa refinery. In: NPRA Plant Automation \& Decision Support Conference. San Antonio, Texas (2004)

13. Chernoff, H.: Locally optimal designs for estimating parameters. Annals of Mathematical Statistics 24, 586-602 (1953)

14. Fedorov, V.V.: Theory of Optimal Experiments, Probability and Mathematical Statistics, vol. 12. Academic Press (1972)

15. Forssell, U., Ljung, L.: Some results on optimal experiment design. Automatica 36(5), 749_ $756(2000)$

16. Gerencsér, L., Hjalmarsson, H.: Adaptive input design in system identification. In: Proc. of the 44th IEEE Conference on Decision and Control and European Control Conference, pp. 4988-4993. Seville, Spain (2005)

17. Gerencsér, L., Hjalmarsson, H.: Identification of ARX systems with non-stationary inputs asymptotic analysis with application to adaptive input design. Automatica 45(3), 623-633 (2009)

18. Hildebrand, R., Gevers, M.: Identification for control: Optimal input design with respect to a worst-case $v$-gap cost function. SIAM J. Control Optim 41(5), 1586-1608 (2003)

19. Hjalmarsson, H.: System identification of complex and structured systems. European Journal of Control 15(4), 275-310 (2009). Plenary address. European Control Conference

20. Hjalmarsson, H., Jansson, H.: Closed loop experiment design for linear time invariant dynamical systems via LMIs. Automatica 44(3), 623-636 (2008)

21. Hjalmarsson, H., Mårtensson, J.: Optimal input design for identification of non-linear systems: Learning from the linear case. In: American Control Conference. New York City, USA (2007)

22. Jansson, H.: Experiment design with applications in identification for control. Doctoral thesis, KTH, Stockholm, Sweden (2004)

23. Jansson, H., Hjalmarsson, H.: Input design via LMIs admitting frequency-wise model specifications in confidence regions. IEEE Transactions on Automatic Control 50(10), 1534-1549 (2005)

24. Ljung, L.: System Identification: Theory for the User, 2nd edition edn. Prentice-Hall, Englewood Cliffs, NJ (1999)

25. Mårtensson, J.: Geometric analysis of stochastic model errors in system identification. Doctoral thesis, KTH, Stockholm, Sweden (2007) 
26. Mårtensson, J., Hjalmarsson, H.: How to make bias and variance errors insensitive to system and model complexity in identification. IEEE Transactions on Automatic Control 56(1), 100$112(2011)$

27. Oppenheim, A., Schafer, R.: Discrete-Time Signal Processing. Prentice-Hall, Englewood Cliffs, NJ (1989)

28. Pronzato, L.: Optimal experimental design and some related control problems. Automatica 44(2), 303-325 (2008)

29. Pukelsheim, F.: Optimal design of experiments. John Wiley (1993)

30. Rantzer, A.: On the Kalman-Yakubovich-Popov lemma. Systems and Control letters 28(1), 7-10 (1996)

31. Rojas, C., Hjalmarsson, H., Gerencsér, L., Mårtensson, J.: Consistent estimation of real NMP zeros in stable LTI systems of arbitrary complexity. In: 15th IFAC Symposium on System Identification, pp. 922-927. Saint-Malo, France (2009)

32. Rojas, C.R.: Robust experiment design. Ph.D. thesis, The University of Newcastle (2008)

33. Rojas, C.R., Barenthin, M., Welsh, J.S., Hjalmarsson, H.: The cost of complexity in identification of FIR systems. In: 17th IFAC World Congress, pp. 11,451-11,456. Seoul, South Korea (2008)

34. Rojas, C.R., Welsh, J.S., Goodwin, G.C., Feuer, A.: Robust optimal experiment design for system identification. Automatica 43(6), 993-1008 (2007)

35. Welsh, J.S., Rojas, C.R.: A scenario based approach to robust experiment design. In: Proceedings of the 15th IFAC Symposium on System Identification (SYSID'09). Saint-Malo, France (2009)

36. Yakubovich, V.A.: Solution of certain matrix inequalities occurring in the theory of automatic control. Docl. Acad. Nauk. SSSR pp. 1304-1307 (1962)

37. Zarrop, M.: Optimal Experiment Design for Dynamic System Identification. Lecture Notes in Control and Information Sciences. Sci. 21. Springer Verlag, Berlin (1979)

38. Zhang, F. (ed.): The Schur Complement and Its Applications, Numerical Methods and Algorithms, vol. 4. Springer (2005) 